Ciência e Natura, Santa Maria v.37 n.3, 2015, Set.- Dez. p. 920 - 933

Revista do Centro de Ciências Naturais e Exatas - UFSM

ISSN impressa: 0100-8307 ISSN on-line: 2179-460X

\title{
CIÊNCIAPNATURA 35旁
}

\section{Modelos de processos difusivos e solução aproximada usando volumes finitos}

\author{
Models of diffusive processes and approximate solution using finite volume
}

\author{
Uilbiran Chaves Santos ${ }^{1}$ e Domingos Boaes Garcia ${ }^{2}$ \\ ${ }^{1}$ Docente, Departamento de matemática - Instituto Federal do Maranhão, MA - Brasil \\ uilchaves28@ifma.edu.br \\ ${ }^{2}$ Docente, Departamento de matemática - Instituto Federal do Maranhão, MA - Brasil \\ domingos.boaes@ifma.edu.br
}

\section{Resumo}

\section{Resumo}

Discutimos, neste trabalho, o papel das equações diferenciais, ordinárias e parciais, na representação matemática de processos reais que envolvam leis de conservação usadas na física, dando enfoque nas equações de difusão que resultam do princípio de conservação de energia. Apresentamos o método dos volumes finitos, uma técnica atual e bastante útil usada na discretização de equações diferenciais parciais parabólicas e hiperbólicas. Aplicamos tal método para encontrar aproximações para a solução da equação do calor transiente bidimensional.

Palavras-chave: Modelos matemáticos, Equações de calor, Método de volumes finitos.

\begin{abstract}
We have discussed in this work the role of differential ordinary and partial equations in mathematical representation of real processes involving conservation laws used in physics, focusing on diffusion equations that result from energy conservation principle. Here we present the finite volume method, a modern technique and widely used in the discretization of partial parabolic and hyperbolic differential equations. We applied this method to find approximations for the transient equation of heat.
\end{abstract}

Keywords: Mathematical models, heat equations, finite volume method. 


\section{Introdução}

De modo geral, um modelo matemático são equações escritas usando símbolos matemáticos que retrata uma visão idealizada da realidade e tem como objetivo o entendimento do fenômeno e possíveis predições do comportamento futuro. Nesta formulação, o que nos interessam são as incógnitas que, em geral são funções de outras variáveis.

Nas aplicações encontramos numerosos fenômenos nos quais a taxa de variação de algumas quantidades presentes no modelo estão relacionadas entre elas e às outras variáveis do modelo. Neste caso as incógnitas do modelo são funções que aparecem sob a operação de diferenciação e na matemática são catalogadas como Equações Diferenciais. As equações diferenciais têm papel importante há mais de quatro séculos, principalmente por que elas representam os princípios básicos da física, que são usados na compreensão da natureza. Por exemplo, são equações diferenciais as representações dos princípios de conservação de massa, de energia e do momento do sistema. Vamos citar alguns exemplos de fenômenos cuja representação matemática envolve taxas de variações.

Exemplo 1. A taxa de decaimento de uma substância radioativa, e a quantidade de radiação que ela emite é, em cada momento, proporcional a sua massa $m(t)$ naquele instante. Desta forma, o processo de desintegração é a equação diferencial ordinária de primeira ordem:

$$
\frac{d m(t)}{d t}=-a m(t)
$$

Nesta equação o parâmetro $a>0$ representa o coeficiente de proporcionalidade, uma característica do material radioativo. $\mathrm{O}$ sinal negativo reflete o decaimento da radioatividade com o passar do tempo.

Exemplo 2. O crescimento de uma população é frequentemente proporcional à população existente. Assim, o modelo simplificado é:

$$
\frac{d p(t)}{d t}=a p(t)
$$

Para melhorar o modelo precisamos considerar as restrições alimentares, a competição entre espécies, etc.

Exemplo 3. Quando um objeto quente, com temperatura $T$, é colocado em um ambiente com temperatura $T_{0}$ (que se presume constante), o objeto esfria a uma taxa que é proporcional à diferença entre sua temperatura no instante $t$ e a temperatura circundante, $T-T_{0}$. Assim, o resfriamento do objeto tem como modelo a lei do resfriamento de Newton:

$$
\frac{d T(t)}{d t}=-a\left[T(x)-T_{0}\right] .
$$

Nos exemplos anteriores as funções incógnitas dependem apenas do tempo e suas taxas de variação são derivadas de funções de uma única variável. São exemplos de Equações Diferenciais Ordinárias, cuja expressão geral é:

$$
\frac{d y(t)}{d t}=f(t, y), \quad y(0)=y_{0}
$$

na qual $f$ representa a relação entre as variáveis e $y_{0}$ é a condição inicial.

No caso de mais de uma variável independente temos as Equações Diferenciais Parciais, mais realísticas quando representamos o mundo real, tridimensional e também a variação no tempo. Por exemplo, de acordo com Lin e Segel (1974), o princípio de conservação de massa numa região do espaço tridimensional, considerando que não há fonte de massa, é representado pela equação diferencial parcial, conhecida como equação da continuidade:

$$
\frac{\partial \rho}{\partial t}+\frac{\partial}{\partial x}\left(\rho u_{x}\right)+\frac{\partial}{\partial y}\left(\rho u_{y}\right)+\frac{\partial}{\partial z}\left(\rho u_{z}\right)=0
$$

Nesta equação a incógnita é a velocidade no ponto $(x, y, z)$ e no instante $t$ e o vetor $u(x, y, z, t)=\left(u_{x}, u_{y}, u_{z}\right)$ representa a velocidade no meio.

O ideal da modelagem matemática é também encontrar métodos matemáticos que forneçam soluções exatas para os modelos, isto é, suas soluções analíticas. Por exemplo, o modelo apresentado no exemplo 1 tem como solução analítica a função $m(t)=m_{0} \exp (-a t)$, onde $m_{0}$ é a massa da substância radioativa no instante inicial. Entretanto em problemas mais complexos, como por exemplo a equação da continuidade em regiões com geometria irregular, dificilmente conhecemos a solução analítica. A dificuldade em obter soluções analíticas favoreceu o desenvolvimento de métodos numéricos para calcular soluções aproximadas. Embora muitos métodos numéricos eficientes já fossem usados antes do século $\mathrm{XX}$, a questão do cálculo numérico restringia bastante sua utilização em modelos mais realísticos. O desenvolvimento dos computadores nos últimos sessenta anos tem ampliado de forma expressiva a gama de problemas que podem ser investigados usando aproximações fornecidas por métodos numéricos. Presenciamos um crescente desenvolvimento de programas computacionais, chamados simuladores em algumas áreas, que tem como modelo sistemas de equações diferenciais que incorporam mais e mais as complexidades dos fenômenos em estudo.

\section{Processos Difusivos: Equações Pa- rabólicas}

Nesta seção usa-se o Princípio da conservação de Energia, na forma de calor, para obter uma equação diferencial que representa matematicamente este princípio. Esta 
é a Equação do Calor, protótipo das equações parabólicas, que são amplamente usadas no estudo de processos difusivos.

De acordo Kreider (1972), para obter a versão bidimensional desta equação, considera-se uma placa homogênea fina

$$
R=\{(x, y) ; 0<x<L \quad \text { e } \quad 0<y<M\},
$$

isolada de modo que nenhum calor flua através de suas faces, conforme Figura 1, isto é, que o fluxo de calor na placa se realiza apenas na direção dos eixos $x$ e $y$. Além disso, suponha que a temperatura na placa seja uniforme em cada seção perpendicular aos eixos coordenados.

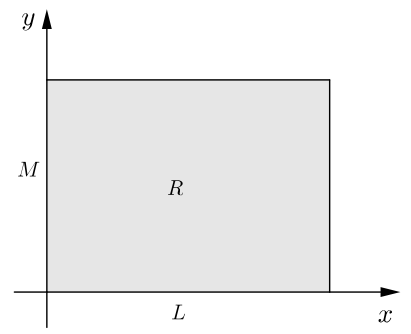

Figura 1: Placa retangular homogênea.

O Princípio da Conservação do calor nos diz que: $A$ quantidade de calor acumulado numa região $R$, por unidade de tempo, é igual à resultante do fluxo de calor que passa pela fronteira de $R$ somado à quantidade de calor gerado no interior de $R$, por unidade de tempo.

Os símbolos matemáticos podem ser usados para reescrever cada uma das três parcelas que estão presentes no balanço acima: termo de acumulação, termo do fluxo e termo de geração. Esta é a forma de obter uma equação matemática que represente um princípio físico.

Detalha-se cada uma das parcelas separadamente considerando uma porção arbitrária da placa $R$, definida por

$$
R_{p}=\{(x, y) / \bar{x} \leq x \leq \bar{x}+\Delta x \quad \text { e } \quad \bar{y} \leq y \leq \bar{y}+\Delta y\},
$$

conforme Figura 2.

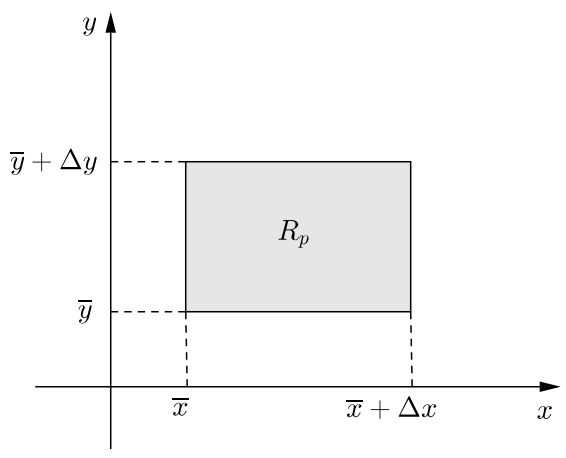

Figura 2: Porção da placa $R$.

A quantidade de calor, $\Delta Q$, que se acumula em qualquer porção de uma placa, é proporcional ao produto de sua massa $m$ pela taxa de variação da temperatura, aqui representada pela função $T(x, y, t)$ :

$$
\Delta Q=c m \frac{\partial T}{\partial t}
$$

para uma constante positiva $c$, conhecida como calor específico do material que compõe o corpo representado matematicamente por $R$. Dessa forma, se $\rho$ designa a densidade do material (massa por unidade de área), então, em razão da equação (1) a quantidade de calor que se acumula em $R_{p}$ por unidade de tempo, é :

$$
\frac{\partial Q}{\partial t}=c \rho \triangle x \Delta y \frac{\partial T}{\partial t}
$$

sendo $\frac{\partial T}{\partial t}$ calculada em algum ponto de $R_{p}$.

Passa-se ao cálculo do fluxo que atravessa a fronteira de $R_{p}$. A lei de Fourier nos diz que o fluxo de calor que passa através de uma superfície é proporcional à derivada normal da temperatura. No caso da placa $R_{p}$, Figura 2, o fluxo de calor se dá nas seções retas localizadas na direção dos eixos coordenados e, portanto, a taxa de variação do fluxo de calor num ponto $(\bar{x}, \bar{y})$, por unidade de área e unidade de tempo pode ser escrito em termos do gradiente da temperatura neste ponto:

$$
-k_{x} \frac{\partial T}{\partial x}-k_{y} \frac{\partial T}{\partial y}
$$

Esta é a Equação Constitutiva que informa como se difunde o calor no material que compõe a placa, e as constantes de proporcionalidades $k_{x}$ e $k_{y}$ são chamadas coeficientes de condutividade térmica, uma característica do material. Sendo $k_{x}$ e $k_{y}$ positivos, e como o fluxo ocorre na direção da diminuição da temperatura, o sinal negativo na equação (3) é necessário. Adotando a convenção de sinais dos eixos $x$ e $y$, o calor que entra em $R_{p}$, na direção $x$ é:

$$
k_{x} \triangle y \frac{\partial T}{\partial x}(\bar{x}, y, t)
$$

e o que sai :

$$
-k_{x} \triangle y \frac{\partial T}{\partial x}(\bar{x}+\triangle x, y, t)
$$

com $\bar{y} \leq y \leq \bar{y}+\Delta y$.

Analogamente, na direção y o calor que entra e que sai é, respectivamente:

$$
k_{y} \triangle x \frac{\partial T}{\partial y}(x, \bar{y}, t) \quad e \quad-k_{y} \triangle x \frac{\partial T}{\partial y}(x, \bar{y}+\triangle x, t),
$$

com $\bar{x} \leq x \leq \bar{x}+\Delta x$. Assim, o fluxo de calor resultante (o calor que entra menos o calor que sai) é:

$$
k_{x} \Delta y\left[\frac{\partial T}{\partial x}(\bar{x}+\Delta x, y, t)-\frac{\partial T}{\partial x}(\bar{x}, y, t)\right]+
$$




$$
k_{y} \Delta x\left[\frac{\partial T}{\partial y}(x, \bar{y}+\Delta y, t)-\frac{\partial T}{\partial y}(x, \bar{y}, t)\right] .
$$

$\mathrm{O}$ terceiro termo do balanço de energia, que representa o calor gerado por unidade de área e de tempo, no interior de $R_{p}$ é escrito em termos da função $f(x, y, t)$. Assim, a quantidade de calor gerada em $R_{p}$, por unidade de tempo, é dada por :

$$
\Delta x \Delta y f(x, y, t) .
$$

Usando as equações (2), (4) e (5) no princípio de conservação de calor, tem-se:

$$
\begin{aligned}
c \rho \frac{\partial T}{\partial t}= & k_{x} \frac{1}{\Delta x}\left[\frac{\partial T}{\partial x}(\bar{x}+\Delta x, y, t)-\frac{\partial T}{\partial x}(\bar{x}, y, t)\right]+ \\
& k_{y} \frac{1}{\Delta y}\left[\frac{\partial T}{\partial y}(x, \bar{y}+\Delta y, t)-\frac{\partial T}{\partial y}(x, \bar{y}, t)\right]+ \\
& f(x, y, t)
\end{aligned}
$$

e passando ao limite quando $\Delta x$ e $\Delta y$ tendem a zero, obtém-se

$$
a^{2} \frac{\partial T}{\partial t}=k_{x} \frac{\partial^{2} T}{\partial x^{2}}+k_{y} \frac{\partial^{2} T}{\partial y^{2}}+f(x, y, t),
$$

onde $c \rho$ foi substituída por $a^{2}$ para ressaltar que ela é positiva. Esta equação é conhecida também como a equação da difusão bidimensional.

De acordo com Cunha (2003), os problemas físicos têm condições de contorno que devem descrever matematicamente o que está acontecendo no contorno do corpo em estudo. Além da razão física, adicionar condições extras à equação diferencial é crucial na descrição adequada do problema, do ponto de vista matemático. Em outras palavras, o conjunto equação diferencial, condições de contorno e condições iniciais, deve ser um problema bem posto: ter uma única solução e esta solução deve depender continuamente dos dados do problema (pequenas pertubações nos dados implicam em pequenas variações da solução).

Através da condição inicial informa-se como é a solução no início do processo em estudo, $t=0$. No caso da equação do calor (6), a condição inicial é uma função que traduz a distribuição da temperatura no tempo inicial:

$$
T(x, y, 0)=T_{0}(x, y), \quad 0 \leq x \leq L \quad \text { e } \quad 0 \leq y \leq M .
$$

As condições de contorno devem ser usadas nas seções retas localizadas nos eixos coordenados, as quais podem ser dos seguintes tipos.

Se a temperatura é conhecida nos extremos, tem-se condições do tipo Dirichlet:

$$
T(0, y, t)=f_{1}(y, t) \text { e } T(L, y, t)=f_{2}(y, t) ;
$$

$$
T(x, 0, t)=g_{1}(x, t) \text { e } T(x, M, t)=g_{2}(x, t),
$$

com $t \geq 0$.

Se o fluxo de calor é conhecido, incluindo o caso de fronteiras isoladas: $h_{1}(y, t)=h_{2}(y, t)=0 \quad$ e $w_{1}(y, t)=w_{2}(y, t)=0$, tem-se Condições de Neumann:

$$
\begin{gathered}
\frac{\partial T}{\partial x}(0, y, t)=h_{1}(y, t) \text { e } \frac{\partial T}{\partial x}(L, y, t)=h_{2}(y, t) ; \\
\frac{\partial T}{\partial y}(x, 0, t)=w_{1}(x, t) \mathrm{e} \frac{\partial T}{\partial y}(x, M, t)=w_{2}(x, t),
\end{gathered}
$$

com $t \geq 0$.

Se a temperatura da vizinhança da fronteira é conhecida, tem-se Condições Mistas. Neste caso, pode-se utilizar a Lei de Fourier para obter as seguintes relações:

$$
\begin{aligned}
& \frac{\partial T}{\partial x}(0, y, t)=\lambda_{1}\left[T(0, y, t)-f_{1}(y, t)\right] ; \\
& \frac{\partial T}{\partial x}(L, y, t)=\lambda_{2}\left[T(L, y, t)-f_{2}(y, t)\right] ; \\
& \frac{\partial T}{\partial y}(x, 0, t)=\mu_{1}[T(x, 0, t)-g 1(x, t)] ; \\
& \frac{\partial T}{\partial y}(x, 0, t)=\mu_{2}\left[T(x, M, t)-g_{2}(x, t)\right],
\end{aligned}
$$

$\operatorname{com} t \geq 0$.

\section{O Método dos Volumes Finitos}

O método dos volumes finitos é um método de discretização que é utilizado para obter a solução numérica de vários tipos de equações diferenciais, a partir da integração da equação diferencial em uma região, chamada volume de controle. Uma característica importante do método dos volumes finitos é que em cada volume discretizado, a grandeza física em questão, por exemplo a massa, obedece à uma lei de conservação, ou seja, sua quantidade permanece conservada a nível discreto. Esta característica torna o método dos volumes finitos muito útil quando se quer modelar problemas para os quais o fluxo é importante, como por exemplo, na mecânica dos fluidos.

O fluxo de uma grandeza, como massa ou energia, é definido pela a quantidade dessa grandeza que atravessa uma região com área $\mathrm{A}$, por unidade de tempo. Em geral são os experimentos que nos informam as características do fluxo da grandeza em estudo. Por exemplo, a lei de Fourier estabelece a relação entre o fluxo de calor e a derivada normal da temperatura, conforme equação (3).

A quantidade de uma grandeza $U$ que atravessa as fronteiras de um volume de controle $V$ por unidade de tempo, é calculada pelo balanço na fronteira, isto é, pela diferença entre o fluxo que entra e o que sai de $V$. Os fluxos, de um modo geral, são classificados conforme de Oliveira Fortuna (2000) em: 
- Fluxos convectivos, que estão associados à velocidade do fluido. Por exemplo, se $U$ for a temperatura, o fluxo convectivo seria o fluxo de calor devido ao escoamento de água quente de uma região mais quente para a região onde a água estivesse mais fria.

- Fluxos difusivos, que são causados pela não-uniformidade da distribuição espacial de $U$. Novamente considerando $U$ como temperatura, surge um fluxo de calor na direção $x$, por exemplo, quando há um gradiente de temperatura presente nessa direção. A componente do fluxo de calor na direção $x$, dada por $k \frac{\partial U}{\partial x}$, onde $k$ é o coeficiente de condutividade térmica do meio, aparece no sentido da temperatura mais alta para a mais baixa. Como vê-se, a natureza do fluxo difusivo é diferente do convectivo, pois mesmo sem movimento pode ocorrer fluxo difusivo.

O resultado do balanço da grandeza que cruza a fronteira de $V$ somado à produção de $U$ em $V$, é proporcional à variação temporal de $U$ dentro do volume de controle.

Dessa forma, considerando apenas fluxo difusivo, pode-se escrever a lei de conservação de uma grandeza $U$ em um volume de controle $V$ :

Taxa de variação temporal elevado de U em $V=$ Entrada de U em $V+$ Produção de U em $V$.

Na expressão acima consideramos produção positiva (geração) ou negativa (sumidouro).

A seguir vamos usar o método dos volumes finitos para obter equações discretizadas associadas a esta expressão, e que serão usadas na obtenção de soluções aproximadas para uma equação diferencial difusiva.

\section{Discretização Conservativa da Lei de Conservação}

Suponha que o fluxo de uma grandeza $U$ seja representado pela função vetorial $\vec{F}$. O modelo matemático que representa uma lei de conservação de $U$, num domínio $\Omega$, é a equação diferencial

$$
U_{t}=\nabla \cdot \vec{F}+Q
$$

onde $Q$ é o termo fonte, que representa a produção de $U$ em $\Omega$.

Como veremos, discretiza-se o domínio, isto é, gerase uma malha que será usada na divisão de $\Omega$ em volumes de controle $\Omega_{j}$.
O método dos volumes finitos consiste em integrar a equação diferencial em cada volume de controle, isto é, transformar (7) na equação diferencial

$$
\int_{\Omega_{j}} \frac{\partial U}{\partial t} d \Omega=\int_{\Omega_{j}} \nabla \cdot \vec{F} d \Omega+\int_{\Omega_{j}} Q d \Omega .
$$

A integral da divergência de um vetor de funções $\nabla . \vec{F}$ no volume de controle $\Omega_{j}$ é, pelo teorema de Gauss, igual à integral de superfície de $\vec{n} . \vec{F}$ :

$$
\int_{\Omega_{j}} \nabla \cdot \vec{F} d \Omega=\oint_{\partial \Omega_{j}} \vec{n} \cdot \vec{F} d s
$$

onde $\partial \Omega_{j}$ é o contorno fechado de $\Omega_{j}$ e $\vec{n}$ o vetor unitário normal a $\partial \Omega_{j}$. Assim, tem-se:

$$
\int_{\Omega_{j}} \frac{\partial U}{\partial t} d \Omega=\oint_{\partial \Omega_{j}} \vec{n} . \vec{F} d s+\int_{\Omega_{j}} Q d \Omega .
$$

Esta é a equação básica do Método dos Volumes Finitos, que tem como uma de suas principais vantagens trabalhar com as componentes dos fluxos sobre o contorno do domínio.

Aproxima-se as integrais da equação (8) para se chegar na forma discretizada da lei de conservação.

Em resumo, o método consiste em 4 etapas:

- Divisão do domínio de análise em volumes de controle finitos;

- Integração da equação diferencial nos volumes de controle;

- Discretização de cada integral de modo a obter um conjunto de equações algébricas;

- Solução do sistema de equações resultantes, empregando métodos numéricos.

\section{Malhas e Volumes de Controle}

Uma malha é um conjunto de linhas que se intersectam em pontos que são os nós. Cada nó da malha pode ser conceptualizado como o ponto representativo do volume de controle que o rodeia. A malha deve abranger todo o domínio físico da solução e os seus nós são os pontos onde a variável dependente, temperatura para o caso da condução de calor, assumirá valores que são a solução da equação discretizada.

Dentre os possíveis tipos de malhas, há malhas estruturadas, que apresentam uma estrutura ou regularidade entre os pontos na distribuição espacial e malhas não-estruturadas, devido à ausência de regularidade na distribuição dos pontos.

Em malhas estruturadas, as fronteiras dos volumes de controle são definidas pelas mediatrizes dos segmentos que unem dois nós consecutivos, conforme Figura 3. 


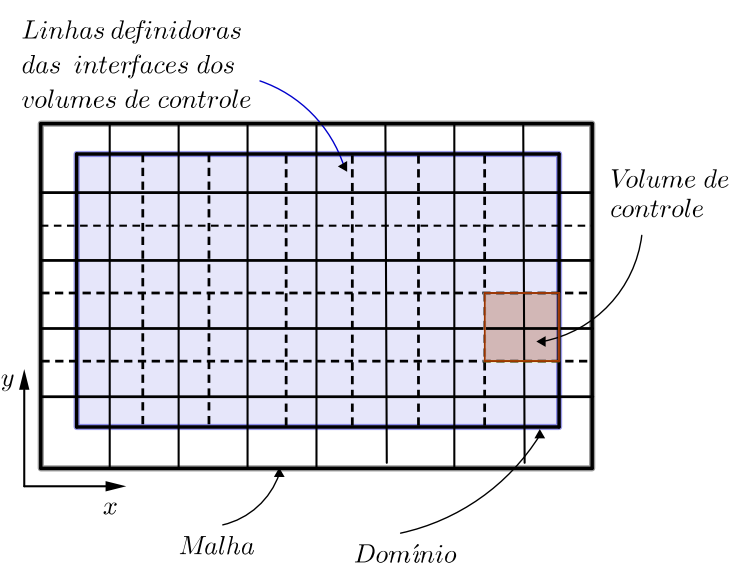

Figura 3: Malha em coordenadas cartesianas (linhas cheias) e volumes de controle (linhas tracejadas).

Apesar dessa figura induzir tal raciocínio, uma malha estruturada não tem necessariamente que ter um espaçamento uniforme. No caso da malha ser uniforme, o nó que representa um determinado volume de controle se localizará no centro geométrico desse volume, tal como se pode observar na Figura 4, que representa um volume de controle interno, isto é, que não possui nenhuma face no contorno do domínio.

A notação usada também é apresentada na Figura 4. As interfaces dos volumes de controle são denotadas por $e, w, n$ e $s$ e suas dimensões são $\Delta x$ na direção do eixo $x$ e $\Delta y$ na direção do eixo $y$. As outras dimensões como por exemplo $\Delta x_{E P}$ ou $\Delta y_{P S}$, são auto-explicativas. Neste caso, em que a malha é uniforme, $\Delta x_{E P}=\Delta x_{P W}=\Delta x$ e $\Delta y_{N P}=\Delta y_{P S}=\Delta y$. As variáveis $q_{e}, q_{w}, q_{n}$ e $q_{s}$ representam os fluxos nas interfaces dos volumes de controle.

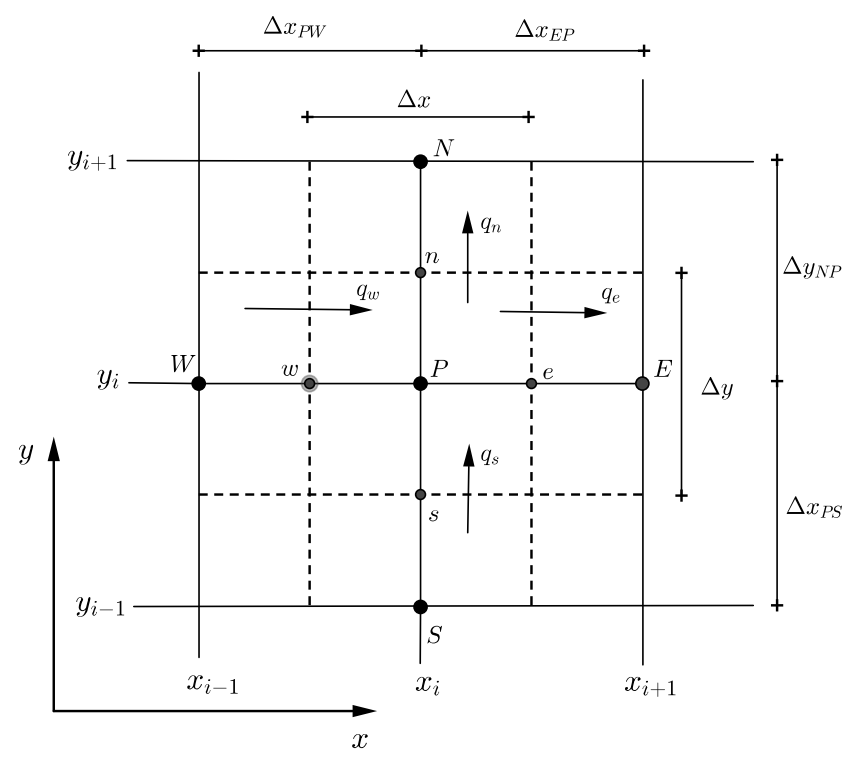

Figura 4: Volume de controle interno
Na próxima seção utiliza-se o método dos volumes finitos na discretização da equação da condução de calor bidimensional utilizando uma malha estruturada com espaçamentos uniformes.

\section{Uma aplicação: condução do calor}

Considere a equação da condução térmica bidimensional, transiente e com termo fonte, deduzida na seção 2. Assim sendo, procura-se a temperatura $T(x, y, t)$ que é governada pela equação (6):

$$
c \rho \frac{\partial T}{\partial t}=k_{x} \frac{\partial^{2} T}{\partial x^{2}}+k_{y} \frac{\partial^{2} T}{\partial y^{2}}+f,
$$

onde $(x, y) \in R$, uma região do plano $(x, y), t \geq 0$, $\rho(x, y, t)>0$ é a densidade do meio, $c(x, y, t)>0$ é o calor específico do material pelo qual o calor é conduzido, $k_{x}(x, y, t)>0$ e $k_{y}(x, y, t)>0$ são os coeficientes de condutividade térmica nas direções $x$ e $y$, respectivamente, e $f(x, y, t)$ o termo fonte.

A equação (9) pode ser rescrita usando o operador divergente:

$$
\frac{\partial}{\partial t}(c \rho T)=\nabla \cdot \vec{F}+f
$$

onde

$$
\vec{F}=k_{x} \frac{\partial T}{\partial x} \vec{i}+k_{y} \frac{\partial T}{\partial y} \vec{j}
$$

denota o fluxo que passa através das faces do volume de controle.

No caso em que $R$ é um retângulo

$$
R=\left\{(x, y) ; 0<x<l_{1} \text { e } 0<y<l_{2}\right\} \text {, }
$$

a malha usada na discretização espacial é:

$x_{i}=\left(\frac{2 i-1}{2}\right) \Delta x \quad i=0: m_{1}, \quad$ onde $\quad\left(m_{1}-1\right) \Delta x=l_{1}$;

$y_{j}=\left(\frac{2 j-1}{2}\right) \Delta y \quad j=0: m_{2}, \quad$ onde $\quad\left(m_{2}-1\right) \Delta y=l_{2}$.

Na variável tempo, toma-se $t_{n}=n \Delta t, n=0,1, \ldots$. Denota-se por $T_{i, j}^{n}$ a aproximação de $T(x, y, t)$ no ponto da malha $\left(x_{i}, y_{j}\right)$ no tempo $t_{n}$, isto é,

$$
T_{i, j}^{n} \cong T\left(x_{i}, y_{j}, t_{n}\right)
$$

A Figura 3 ilustra os volumes de controle definidos pela malha.

Integrando a equação (10) no volume de controle $V$ de referência e no intervalo de tempo $\left[t_{n}, t_{n+1}\right]$, obtém-se:

$$
\int_{t_{n}}^{t_{n+1}} \int_{V} \frac{\partial(c \rho T)}{\partial t} d V d t=\int_{t_{n}}^{t_{n+1}} \int_{V} \nabla \cdot \vec{F} d V d t+
$$




$$
\int_{t_{n}}^{t_{n+1}} \int_{V} f d V d t
$$

Considerando $c$ e $\rho$ constantes e supondo suavidade de $\frac{\partial T}{\partial t}$, pode-se inverter a ordem de integração do lado esquerdo da equação (12):

$$
\begin{aligned}
c \rho \int_{V} \int_{t_{n}}^{t_{n+1}} \frac{\partial T}{\partial t} d t d V= & \int_{t_{n}}^{t_{n+1}} \int_{V} \nabla \cdot \vec{F} d V d t+ \\
& \int_{t_{n}}^{t_{n+1}} \int_{V} f d V d t .
\end{aligned}
$$

\section{Desenvolvimento do termo transi- ente}

Usando o teorema fundamental do cálculo, tem-se:

$$
\begin{aligned}
c \rho \int_{V} \int_{t_{n}}^{t_{n+1}} \frac{\partial T}{\partial t} d t d V & =c \rho \int_{V}\left(T^{n+1}-T^{n}\right) d V \\
& \cong c \rho\left(T_{P}^{n+1}-T_{P}^{n}\right) \Delta V,
\end{aligned}
$$

onde na última passagem usa-se a fórmula de integração dos retângulos (ponto médio).

\section{Desenvolvimento do termo diver- gente}

Aplicando o teorema da Divergência de Gauss, transformase a integral de volume em uma integral de linha:

$$
\int_{t_{n}}^{t_{n+1}} \int_{V} \nabla \cdot \vec{F} d V d t=\int_{t_{n}}^{t_{n+1}} \oint_{S} \vec{n} \cdot \vec{F} d S
$$

sendo $S$ o contorno fechado do volume $V$, e $\vec{n}$ um vetor unitário normal a $S$ que aponta para fora de $S$. Por outro lado:

$$
\oint_{S} \vec{n} \cdot \vec{F} d S=\sum_{q} \int_{S_{q}} \vec{n} \cdot \vec{F} d S
$$

onde $q$ representa as interfaces $e, w, n$ e $s$, conforme mostra a Figura 4. Desta forma, $\vec{n}=(1,0)$ na interface $e$, $\vec{n}=(-1,0)$ na interface $w, \vec{n}=(0,1)$ na interface $n$ e $\vec{n}=(0,-1)$ na interface $s$. Assim, pela equação (11):

$$
\begin{aligned}
\oint_{S} \vec{n} \cdot \vec{F} d S= & \int_{S_{e}} k_{x} \frac{\partial T}{\partial x} d S-\int_{S_{w}} k_{x} \frac{\partial T}{\partial x} d S+ \\
& \int_{S_{n}} k_{y} \frac{\partial T}{\partial y} d S-\int_{S_{S}} k_{y} \frac{\partial T}{\partial y} d S .
\end{aligned}
$$

Utilizando a regra do ponto médio, aproximamos cada uma das integrais na equação (15) pelo produto do integrando no ponto médio de cada interface por seu comprimento. Por exemplo:

$$
\left.\int_{S e} k_{x} \frac{\partial T}{\partial x} d S \cong \Delta y\left(k_{x} \frac{\partial T}{\partial x}\right)\right|_{e}
$$

onde $\left.\left(k_{x} \frac{\partial T}{\partial x}\right)\right|_{e}$ é o valor do fluxo no ponto médio da interface $e$. Assim,

$$
\begin{aligned}
\oint_{S} \vec{n} \vec{F} d S \cong & {\left[\left.\left(k_{x} \frac{\partial T}{\partial x}\right)\right|_{e}-\left.\left(k_{x} \frac{\partial T}{\partial x}\right)\right|_{w}\right] \Delta y+} \\
& {\left[\left.\left(k_{y} \frac{\partial T}{\partial y}\right)\right|_{n}-\left.\left(k_{y} \frac{\partial T}{\partial y}\right)\right|_{S}\right] \Delta x . }
\end{aligned}
$$

Considerando $k_{x}$ e $k_{y}$ constantes e aproximando os termos difusivos por diferenças centrais,

$$
\left.\left(k_{x} \frac{\partial T}{\partial x}\right)\right|_{e} \cong k_{x}\left(\frac{T_{E}-T_{P}}{\Delta x}\right)
$$

com erro de ordem $(\Delta x)^{2}$, tem-se

$$
\begin{aligned}
\oint_{S} \vec{n} \vec{F} d S \cong & {\left[k_{x}\left(\frac{T_{E}-T_{P}}{\Delta x}\right)-k_{x}\left(\frac{T_{P}-T_{W}}{\Delta x}\right)\right] \Delta y+} \\
& {\left[k_{y}\left(\frac{T_{N}-T_{P}}{\Delta y}\right)-k_{y}\left(\frac{T_{P}-T_{S}}{\Delta y}\right)\right] \Delta x,(17) }
\end{aligned}
$$

com erro de truncamento de ordem $O\left[(\Delta x)^{2}+(\Delta y)^{2}\right]$. Dessa forma, o lado direito da equação (17) fica:

$$
\begin{aligned}
F(T(t))= & -2\left(k_{x} \frac{\Delta y}{\Delta x}+k_{y} \frac{\Delta x}{\Delta y}\right) T_{P}(t)+\left(k_{x} \frac{\Delta y}{\Delta x}\right) T_{E}(t)+ \\
& \left(k_{x} \frac{\Delta y}{\Delta x}\right) T_{W}(t)+\left(k_{y} \frac{\Delta x}{\Delta y}\right) T_{N}(t)+ \\
& \left(k_{y} \frac{\Delta x}{\Delta y}\right) T_{S}(t) .
\end{aligned}
$$

Assim, obtém-se a seguinte aproximação para a equação (14):

$$
\int_{t_{n}}^{t_{n+1}} \int_{V} \nabla \cdot \vec{F} d V d t \cong \int_{t_{n}}^{t_{n+1}} F(T(t)) d t,
$$

com erro de truncamento de ordem $O\left[(\Delta x)^{2}+(\Delta y)^{2}\right]$.

O lado direito da equação (18) só pode ser integrado se for feita alguma aproximação temporal para $F$, utilizando as regras usuais de integração numérica.

Considera-se as seguintes aproximações:

$$
\int_{t_{n}}^{t_{n+1}} F(T(t)) d t \cong F\left(T^{n}\right) \Delta t,
$$


com erro de ordem $\Delta t$,

$$
\int_{t_{n}}^{t_{n+1}} F(T(t)) d t \cong F\left(T^{n+1}\right) \Delta t,
$$

com erro da ordem de $\Delta t$,

$$
\int_{t_{n}}^{t_{n+1}} F(T(t)) d t \cong\left[\frac{F\left(T^{n+1}\right)+F\left(T^{n}\right)}{2}\right] \Delta t,
$$

com erro da ordem de $(\Delta t)^{2}$, pois de acordo com de Oliveira Fortuna (2000), esta aproximação é a regra dos trapézios.

As três formulações anteriores podem ser generalizadas pela expressão

$$
\int_{t_{n}}^{t_{n+1}} F(T(t)) d t \cong\left[\theta F\left(T^{n+1}\right)+(1-\theta) F\left(T^{n}\right)\right] \Delta t,
$$

em que $\theta$ é uma constante real que varia entre 0 e 1 .

\section{Equação (9) discretizada}

Inicialmente, discretiza-se o termo fonte usando a metodologia da seção anterior, isto é:

$$
\begin{aligned}
\int_{t_{n}}^{t_{n+1}} \int_{V} f d V d t & \cong \int_{t_{n}}^{t_{n+1}} f_{P} \Delta V d t \\
& \cong\left[\theta f_{P}^{n+1}+(1-\theta) f_{P}^{n}\right] \Delta V \Delta t,
\end{aligned}
$$

onde $\theta$ varia entre 0 e 1 e $f_{P}$ é valor de $f$ no centro do volume de controle $V$ de referência, conforme Figura 4 .

Reunindo todos os termos da equação (13), na forma discretizada, obtém-se:

$$
\begin{aligned}
c \rho\left(T_{P}^{n+1}-T_{P}^{n}\right) \Delta V= & {\left[\theta F\left(T^{n+1}\right)+(1-\theta) F\left(T^{n}\right)\right] \Delta t+} \\
& {\left[\theta f_{P}^{n+1}+(1-\theta) f_{P}^{n}\right] \Delta V \Delta t . }
\end{aligned}
$$

Dividindo por $\Delta V \Delta t$, tem-se:

$$
\begin{aligned}
\frac{T_{P}^{n+1}-T_{P}^{n}}{\Delta t}= & \frac{1}{c \rho}\left[\frac{\theta F\left(T^{n+1}\right)+(1-\theta) F\left(T^{n}\right)}{\Delta V}\right]+ \\
& \frac{1}{c \rho}\left[\theta f_{P}^{n+1}+(1-\theta) f_{P}^{n}\right],
\end{aligned}
$$

que é uma forma discretizada da equação (9), utilizando o método dos volumes finitos.

\section{Formulações Explícita, Implícita e de Crank-Nicolson}

De acordo com os valores assumidos por $\theta$ na equação (22), resulta nas formas explícita implícita e de CrankNicolson para a equação (9). A seguir detalha-se estes métodos.

\subsection{O Método Explícito}

Fazendo $\theta=0$ em (22), o método de discretização é o Método Explicito definido por

$$
\frac{T_{P}^{n+1}-T_{P}^{n}}{\Delta t}=\frac{1}{c \rho}\left[\frac{F\left(T^{n}\right)}{\Delta V}+f_{P}^{n}\right] .
$$

Levando em conta as expressões dos erros das discretizações no espaço, equação (17), e no tempo, equação (19), verifica-se que o erro de truncamento da aproximação definida por (23) é de ordem $O\left[\Delta t+(\Delta x)^{2}+(\Delta y)^{2}\right]$.

Com este método, o valor de $T_{P}$ no instante $t_{n+1}, T_{P}^{n+1}$, pode ser encontrado explicitamente em função dos valores da temperatura em $P$ e nos nós vizinhos no instante $t_{n}$.

Substituindo os pontos nodais de (23) pelos seus respectivos índices, definidos na Figura 4, obtém-se:

$$
\begin{aligned}
& \frac{T_{i, j}^{n+1}-T_{i, j}^{n}}{\Delta t}= \\
& \frac{1}{c \rho}\left[k_{x} \frac{T_{i+1, j}^{n}-2 T_{i, j}^{n}+T_{i-1, j}^{n}}{\Delta x^{2}}+k_{y} \frac{T_{i, j+1}^{n}-2 T_{i, j}^{n}+T_{i, j-1}^{n}}{\Delta y^{2}}\right]+ \\
& \frac{1}{c \rho} f_{i, j^{\prime}}^{n}
\end{aligned}
$$

onde $T_{i, j}^{n} \cong T\left(x_{i}, y_{j}, t_{n}\right), i=2:\left(m_{1}-1\right), j=2:\left(m_{2}-1\right)$ e $n=0,1, \ldots$.

Explicitando $T_{i, j}^{n+1}$ em função dos demais termos, resulta:

$$
\begin{aligned}
T_{i, j}^{n+1}= & {[1-2(\alpha+\lambda)] T_{i, j}^{n}+} \\
& \alpha\left(T_{i+1, j}^{n}+T_{i-1, j}^{n}\right)+\lambda\left(T_{i, j+1}^{n}+T_{i, j-1}^{n}\right)+ \\
& \frac{\Delta t}{c \rho} f_{i, j^{\prime}}^{n}
\end{aligned}
$$

$\operatorname{com} \alpha=\frac{\Delta t}{c \rho} \frac{k_{x}}{\Delta x^{2}} \quad$ e $\quad \lambda=\frac{\Delta t}{c \rho} \frac{k_{y}}{\Delta y^{2}}$.

Na Figura 5, assinala-se os pontos envolvidos em cada passo do processo. Designa-se por " $\times$ " os pontos nos quais $T$ é conhecida e usada para as aproximações nos pontos assinalados por "o".
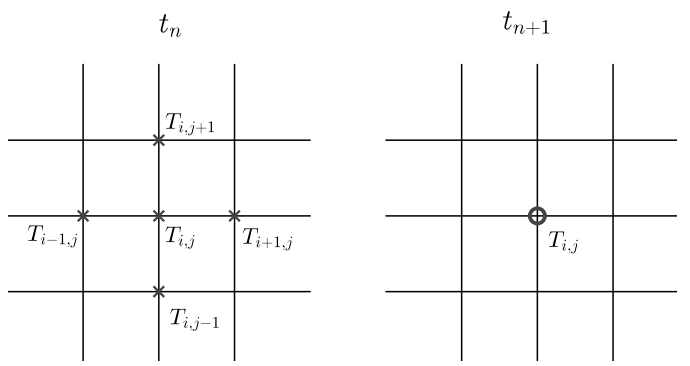

Figura 5: Esquema para o Método Explícito. 
A precisão da aproximação obtida na equação (24) pode ser melhorada com o refinamento da malha, isto é, pela diminuição dos valores de $\Delta x, \Delta y$ e $\Delta t$, uma vez que o erro é de ordem $O\left[\Delta t+(\Delta x)^{2}+(\Delta y)^{2}\right]$. Neste caso, o número de pontos nodais aumenta com o decréscimo de $\Delta x$ e $\Delta y$, e o número de intervalos de tempo necessários para calcular a aproximação até o tempo final também aumenta com a diminuição de $\Delta t$. Logo, o tempo de computação aumenta com o refinamento da malha.

Uma vez escolhidos $\Delta x$ e $\Delta y$, o valor de $\Delta t$ nem sempre pode ser escolhido arbitrariamente. De fato, ele é determinado pelas exigências da estabilidade.

Segundo de Oliveira Fortuna (2000), uma maneira de analisar a estabilidade é escrevendo os valores $T_{i, j}^{n}=T\left(x_{i}, y_{j}, t_{n}\right)$ na forma

$$
T_{i, j}^{n}=\Phi^{n} e^{I Q x_{i}} e^{I R y_{j}},
$$

em que $I=\sqrt{-1}, \Phi^{n}$ sua amplitude no instante $n, Q$ e $R$ os números de onda nas direções $x$ e $y$, respectivamente.

Para que $T_{i, j}$ não aumente sem limites, nenhuma das amplitudes $\Phi$ na equação (25) pode crescer arbitrariamente. "Esta técnica é conhecida como análise de estabilidade de Von Neumann, de acordo com Thomas (1995)".

Como exemplo de aplicação, vamos obter o critério de estabilidade para a discretização da equação (24), no caso particular em que $f(x, y, t)=0$.

Substitui-se cada termo da equação (24) usando a equação (25), isto é, insere-se

$$
\begin{aligned}
T_{i, j}^{n+1} & =\Phi^{n+1} e^{I Q x_{i}} e^{I R y_{j}} \\
T_{i \pm 1, j}^{n} & =\Phi^{n} e^{I Q\left(x_{i} \pm \Delta x\right)} e^{I R y_{j}} \\
T_{i, j \pm 1}^{n} & =\Phi^{n} e^{I Q x_{i}} e^{I R\left(y_{j} \pm \Delta y\right)} .
\end{aligned}
$$

na equação (24). Assim, obtém-se:

$$
\begin{gathered}
\Phi^{n+1} e^{I Q x_{i}} e^{I R y_{j}}=[1-2(\alpha+\lambda)] \Phi^{n} e^{I Q x_{i}} e^{I R y_{j}}+ \\
\alpha \Phi^{n}\left[e^{I Q\left(x_{i}+\Delta x\right)} e^{I R y_{j}}+e^{I Q\left(x_{i}-\Delta x\right)} e^{I R y_{j}}\right]+ \\
\lambda \Phi^{n}\left[e^{I Q x_{i}} e^{I R\left(y_{j}+\Delta y\right)}+e^{I Q x_{i}} e^{I R\left(y_{j}-\Delta y\right)}\right]
\end{gathered}
$$

Divide-se os dois lados dessa equação pelo fator comum $e^{I Q x_{i}} e^{I R y_{j}}$ e agrupa-se os termos, obtendo

$$
\begin{aligned}
\Phi^{n+1}= & \Phi^{n}\left[1-2(\alpha+\lambda)+\alpha\left(e^{I Q x_{i}}+e^{-I Q x_{i}}\right)\right]+ \\
& \Phi^{n} \lambda\left[\left(e^{I R y_{j}}+e^{-I R y_{j}}\right)\right]
\end{aligned}
$$

Os termos que envolvem as exponenciais complexas podem ser simplificadas por meio das identidades

$$
\begin{gathered}
e^{I Q x_{i}}+e^{-I Q x_{i}}=2 \cos (Q \Delta x) \\
e^{I R y_{j}}+e^{-I R y_{j}}=2 \cos (R \Delta y)
\end{gathered}
$$

que substituídas em (26), fatorando os termos comuns, fornecem

$$
\begin{aligned}
\Phi^{n+1} & =\Phi^{n}[1+2 \alpha(\cos (Q \Delta x)-1)+2 \lambda(\cos (R \Delta y)-1)] \\
& =G \Phi^{n} .
\end{aligned}
$$

O termo $G$,

$$
G=1+2 \alpha(\cos (Q \Delta x)-1)+2 \lambda(\cos (R \Delta y)-1)
$$

é conhecido como fator de amplificação, pois ele controla a amplificação ou atenuação de $\Phi^{n}$.

Para que a amplitude $\Phi^{n}$ não aumente a cada passo no tempo, a condição

$$
\left|\frac{\Phi^{n+1}}{\Phi^{n}}\right|=|G| \leq 1
$$

deve ser satisfeita. Isso implica que $\alpha$ e $\lambda$ devem satisfazer à inequação

$$
\begin{aligned}
-1 \leq & 1+2 \alpha(\cos (Q \Delta x)-1)+ \\
& 2 \lambda(\cos (R \Delta y)-1) \leq 1
\end{aligned}
$$

Em função dos valores máximos e mínimos de $\cos (Q \Delta x)$ e $\cos (R \Delta y)$, o lado direito da equação (27) é satisfeito para quaisquer $Q \Delta x, R \Delta y, \alpha$ e $\lambda$. Já para o lado esquerdo, resulta, para os valores mínimos de $\cos (Q \Delta x)-1=-2$ e $\cos (R \Delta y)-1=-2$ :

$$
1-4(\alpha+\lambda) \geq-1
$$

Lembrando que $\alpha=\frac{\Delta t}{c \rho} \frac{k_{x}}{\Delta x^{2}}$ e $\lambda=\frac{\Delta t}{c \rho} \frac{k_{y}}{\Delta y^{2}}$, a desigualdade (28) será:

$$
\frac{\Delta t}{c \rho} \frac{k_{x}}{\Delta x^{2}}+\frac{\Delta t}{c \rho} \frac{k_{y}}{\Delta y^{2}} \leq \frac{1}{2}
$$

ou seja, o passo no tempo deve satisfazer a condição

$$
\Delta t \leq \frac{c \rho}{2\left[k_{x}(\Delta x)^{-2}+k_{y}(\Delta y)^{-2}\right]} .
$$

Este é o critério de estabilidade para o método explícito (24).

Em resumo, a desigualdade (29) estabelece a relação entre o passo na discretização do tempo, $\Delta t$, e os espaçamentos na discretização em $x$ e $y, \Delta x$ e $\Delta y$ respectivamente, de modo que o esquema numérico associado à discretização (24) seja estável.

Quando a estabilidade de um método depende de uma relação entre os tamanhos dos passos utilizados na discretização das variáveis independentes da equação, dizemos que ele é condicionalmente estável. Assim, a condição (24) está nos dizendo que o Método Explícito para a equação do calor (9) é condicionalmente estável. Observe que esta condição pode ser restritiva. Por exemplo, se $c \rho=1, k_{x}=k_{y}=5$ e $\Delta x=\Delta y=10^{-2}$, a estabilidade estará garantida se $\Delta t \leq \frac{1}{2} 10^{-5}$. 


\subsection{O Método Implícito}

Para $\theta=1$ na equação (22), o método de discretização é o Método Implícito. Com este método, o valor da temperatura em cada instante $t_{n+1}, T_{P}^{n+1}$, pode ser calculado implicitamente em função de alguns valores temperatura $T$ no mesmo intervalo de tempo, conforme a equação

$$
\frac{T_{P}^{n+1}-T_{P}^{n}}{\Delta t}=\frac{1}{c \rho}\left[\frac{F\left(T^{n+1}\right)}{\Delta V}+f_{P}^{n+1}\right] .
$$

Como o erro de truncamento na discretização da equação (30) é a soma dos erros de truncamento das discretizações (17) e (20) utilizadas, o erro do Método Implícito é de ordem $O\left[\Delta t+(\Delta x)^{2}+(\Delta y)^{2}\right]$.

Substituindo os pontos nodais pelos seus índices, obtém-se a equação que define o Método Implícito:

$$
\frac{T_{i, j}^{n+1}-T_{i, j}^{n}}{\Delta t}
$$

$\frac{1}{c \rho}\left[k_{x} \frac{T_{i+1, j}^{n+1}-2 T_{i, j}^{n+1}+T_{i-1, j}^{n+1}}{\Delta x^{2}}+k_{y} \frac{T_{i, j+1}^{n+1}-2 T_{i, j}^{n+1}+T_{i, j-1}^{n+1}}{\Delta y^{2}}\right]$

$$
=\frac{1}{c \rho} f_{i, j}^{n+1}
$$

com $i=2:\left(m_{1}-1\right), j=2:\left(m_{2}-1\right)$ e $n=0,1, \ldots$.

Separando as aproximações conhecidas, as do nível de tempo $t_{n}$, das não conhecidas, as do nível de tempo $t_{n+1}$, obtém-se:

$\lambda T_{i, j-1}^{n+1}+\alpha T_{i-1, j}^{n+1}+[1-2(\alpha+\lambda)] T_{i, j}^{n+1}+\alpha T_{i+1, j}^{n+1}+\lambda T_{i, j+1}^{n+1}$

$=T_{i, j}^{n}+\frac{\Delta t}{c \rho} f_{i, j}^{n+1}$,

$\operatorname{com} \alpha=\frac{\Delta t}{c \rho} \frac{k_{x}}{\Delta x^{2}} \quad$ e $\quad \lambda=\frac{\Delta t}{c \rho} \frac{k_{y}}{\Delta y^{2}}$.

Assim, resolve-se um sistema pentadiagonal a cada nível de tempo. O aumento do custo para resolver o sistema é compensado pelo fato do Método Implícito ser incondicionalmente estável, como mostra-se a seguir.

Na discretização pelo Método Implícito, aparecem aproximações das soluções nos pontos da malha $\left(x_{i}, y_{j}, t_{n+1}\right), \quad\left(x_{i-1}, y_{j}, t_{n+1}\right), \quad\left(x_{i}, y_{j}, t_{n}\right),\left(x_{i+1}, y_{j}, t_{n+1}\right)$, $\left(x_{i}, y_{j-1}, t_{n+1}\right)$ e $\left(x_{i}, y_{j+1}, t_{n+1}\right)$, conforme Figura 6 , onde " $x$ " representa os pontos nos quais $T$ é conhecida e usada para as aproximações nos pontos assinalados por "o".

Com relação à estabilidade do Método Implícito, procedendo de modo análogo à subseção anterior, isto é, utilizando a componente de Fourier dada pela equação (25) e a análise de von Neumann, verifica-se que o fator de amplificação do Método Implícito, na ausência de termo fonte, aplicado à equação (31) é dado por

$$
G=\frac{1}{1-2 \alpha(\cos (Q \Delta x)-1)-2 \lambda(\cos (R \Delta y)-1)},
$$
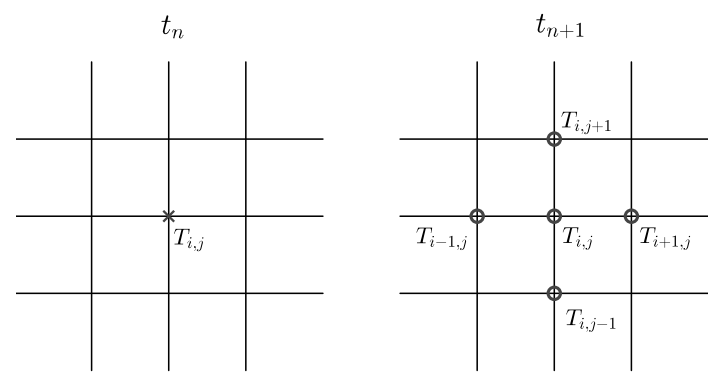

Figura 6: Esquema para o Método Implícito.

em que $\alpha=\frac{\Delta t}{c \rho} \frac{k_{x}}{\Delta x^{2}}$ e $\lambda=\frac{\Delta t}{c \rho} \frac{k_{y}}{\Delta y^{2}}, Q$ e $R$ são os números de ondas nas direções $x$ e $y$, respectivamente.

Em função dos valores máximos e mínimos de $\cos (Q \Delta x)$ e $\cos (R \Delta y)$, a inequação $|G| \leq 1$ é sempre satisfeita. Assim, conclui-se que o Método Implícito é incondicionalmente estável, ou seja, o método permanece estável para qualquer escolha de $\Delta x, \Delta y$ e $\Delta t$.

Dessa forma, uma vez que valores maiores de $\Delta t$ podem ser usados com o Método Implícito, os tempos de cálculo podem ser reduzidos, com pouca perda de precisão. Todavia, para poder maximizar a precisão, $\Delta t$ deverá ser suficientemente pequeno.

\subsection{O Método de Crank-Nicolson}

Fazendo $\theta=\frac{1}{2}$ na equação (22) chega-se ao Método de Crank-Nicolson, no qual os valores da temperatura são definidos por uma média entre os valores das temperaturas nos instantes $t_{n}$ e $t_{n+1}$. Assim sendo, a equação (22) toma a forma:

$$
\frac{T_{P}^{n+1}-T_{P}^{n}}{\Delta t}=\frac{1}{2 c \rho}\left[\frac{F\left(T^{n+1}\right)+F\left(T^{n}\right)}{\Delta V}+f_{P}^{n+1}+f_{P}^{n}\right],
$$

com erro de truncamento de ordem $O\left[\left(\Delta t^{2}\right)+(\Delta x)^{2}+(\Delta y)^{2}\right]$, que é a soma dos erros das discretizações (17) e (21).

Com a substituição dos pontos nodais pelos seus índices, o Método de Crank-Nicolson tem a forma

$\frac{T_{i, j}^{n+1}-T_{i, j}^{n}}{\Delta t}$

$\frac{1}{2 c \rho}\left[k_{x} \frac{T_{i+1, j}^{n+1}-2 T_{i, j}^{n+1}+T_{i-1, j}^{n+1}}{\Delta x^{2}}+k_{y} \frac{T_{i, j+1}^{n+1}-2 T_{i, j}^{n+1}+T_{i, j-1}^{n+1}}{\Delta y^{2}}\right]$

$-\frac{1}{2 c \rho}\left[k_{x} \frac{T_{i+1, j}^{n}-2 T_{i, j}^{n}+T_{i-1, j}^{n}}{\Delta x^{2}}+k_{y} \frac{T_{i, j+1}^{n}-2 T_{i, j}^{n}+T_{i, j-1}^{n}}{\Delta y^{2}}\right]$

$=\frac{1}{2 c \rho} f_{i, j}^{n+\frac{1}{2}}$,

com $i=2:\left(m_{1}-1\right), j=2:\left(m_{2}-1\right)$ e $n=0,1, \ldots$ 
Colocando $T^{n+1}$ em função dos demais termos, temse:

$$
\begin{aligned}
& \lambda T_{i, j-1}^{n+1}+\alpha T_{i-1, j}^{n+1}+2[1-(\alpha+\lambda)] T_{i, j}^{n+1}+\alpha T_{i+1, j}^{n+1}+ \\
& \lambda T_{i, j+1}^{n+1}=\alpha T_{i-1, j}^{n}+\lambda T_{i, j-1}^{n}+2[1-(\alpha+\lambda)] T_{i, j}^{n}+ \\
& \alpha T_{i+1, j}^{n}+\lambda T_{i, j+1}^{n}+\frac{\Delta t}{c \rho} f_{i, j}^{n+\frac{1}{2}}
\end{aligned}
$$

$\operatorname{com} \alpha=\frac{\Delta t}{c \rho} \frac{k_{x}}{\Delta x^{2}} \quad$ e $\quad \lambda=\frac{\Delta t}{c \rho} \frac{k_{y}}{\Delta y^{2}}$.

Na equação do Método de Crank-Nicolson (32), aparecem valores das aproximações em dez pontos da malha, como ilustrado na Figura 7, onde " $x$ " representa os pontos nos quais $T$ é conhecida e usada para as aproximações nos pontos assinalados por "o". Cinco destes pontos são conhecidos, pois correspondem às aproximações no nível de tempo $t_{n}$. Os outros cinco valores são calculados ao se resolver um sistema pentadiagonal. Por isso, o Método de Crank-Nicolson também é implícito. De acordo com Thomas (1995), é possível mostrar que o método de Crank-Nicolson é incondicionalmente estável.
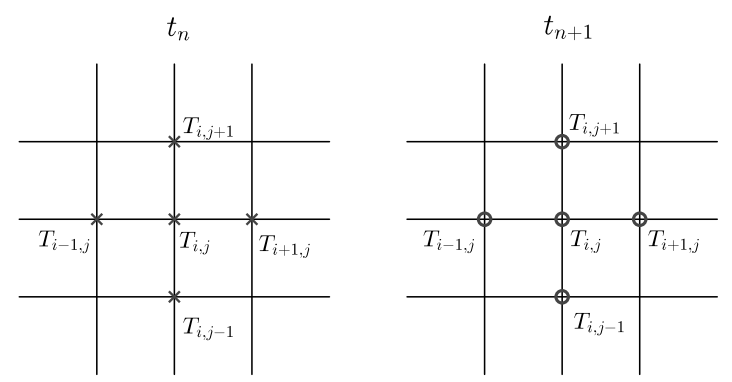

Figura 7: Esquema para o Método Crank-Nicolson.

\section{Inclusão das Condições de Con- torno na Discretização}

A equação (22) representa a equação discretizada para um volume de controle interno. Para se obter o sistema de equações algébricas completo, é também necessário obter as equações para os volumes que estão na fronteira.

Para isso, existem várias alternativas de implementação das condições de contorno do problema; os mais comuns são discretização com meio volume, volumes fictícios e balanços para volume de fronteira, conforme Maliska (2004).

Neste texto, apresenta-se apenas a técnica de balanço para volumes de fronteira. Este é o procedimento considerado adequado por vários autores, conforme Maliska (2004).

Segundo Sperandio et al. (2003), esta técnica consiste em integrar, no espaço e no tempo a equação diferencial também nos volumes de fronteira, da mesma forma realizada para os volumes internos, respeitando as condições de contorno impostas. Assim, as condições de contorno ficam embutidas nas equações para os volumes de fronteira.

A seguir detalha-se o procedimento no caso do Método Explícito. De forma análoga obtém-se as equações discretizadas, referentes aos volumes na fronteira, nos Métodos Implícitos e Crank-Nicolson.

Dessa forma, integrando a equação (9) no volume de referência, na fronteira, conforme Figura 8,

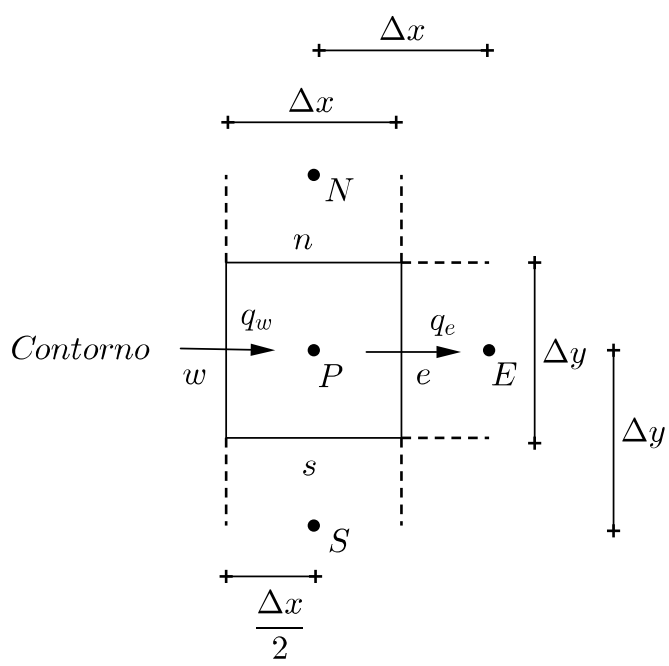

Figura 8: Volume de fronteira-uma face na fronteira

tem-se a seguinte equação:

$$
\frac{T_{P}^{n+1}-T_{P}^{n}}{\Delta t}=\frac{1}{c \rho}\left[\frac{\bar{F}\left(T^{n}\right)}{\Delta V}+\tilde{f}_{P}^{n}\right],
$$

onde

$$
\begin{aligned}
\bar{F}(T(t))= & {\left[\left.\left(k_{x} \frac{\partial T}{\partial x}\right)\right|_{e}-\left.\left(k_{x} \frac{\partial T}{\partial x}\right)\right|_{c w}\right] \Delta y+} \\
& {\left[\left.\left(k_{y} \frac{\partial T}{\partial y}\right)\right|_{n}-\left.\left(k_{y} \frac{\partial T}{\partial y}\right)\right|_{s}\right] \Delta x }
\end{aligned}
$$

sendo $\left.\left(k_{x} \frac{\partial T}{\partial x}\right)\right|_{c w}$ o fluxo no contorno $w$.

As derivadas que não estão na fronteira são avaliadas como na equação (16), e as que estão na fronteira são modificadas, ficando

$$
\left.\left(k_{x} \frac{\partial T}{\partial x}\right)\right|_{c w}=k_{x}\left(\frac{T_{P}-T_{c w}}{\Delta x / 2}\right) .
$$

Substituindo na equação (33) os pontos nodais pelos seus índices, obtém-se:

$$
T_{1, j}^{n+1}=(1-3 \alpha-2 \lambda) T_{1, j}^{n}+\alpha\left(T_{2, j}^{n}+2 T_{0, j}^{n}\right)+
$$




$$
\lambda\left(T_{1, j+1}^{n}+T_{1, j-1}^{n}\right)+\frac{\Delta t}{c \rho} f_{1, j}^{n}
$$

$\operatorname{com} j=2:\left(m_{2}-2\right), \alpha=\frac{\Delta t}{c \rho} \frac{k_{x}}{\Delta x^{2}} \quad$ e $\quad \lambda=\frac{\Delta t}{c \rho} \frac{k_{y}}{\Delta y^{2}}$.

Esta é a discretização da equação (9), utilizando volumes de fronteira, com uma face na fronteira, no caso, face $w$.

Para volumes de fronteira com uma face em $e$, ou uma face em $n$, ou uma face em $s$, considerando $\alpha$ e $\lambda$ como definidos na equação (34), as discretizações são respectivamente:

- Fronteira a Leste: face $e$

$$
\begin{aligned}
T_{m_{1}-1, j}^{n+1}= & (1-3 \alpha-2 \lambda) T_{m_{1}-1, j}^{n}+ \\
& \alpha\left(2 T_{m_{1}-\frac{1}{2}, j}^{n}+T_{m_{1}-2, j}^{n}\right)+ \\
& \lambda\left(T_{m_{1}-1, j+1}^{n}+T_{m_{1}-1, j-1}^{n}\right)+ \\
& \frac{\Delta t}{c \rho} f_{m_{1}-1, j}^{n}
\end{aligned}
$$

$\operatorname{com} j=2:\left(m_{2}-2\right)$.

- Fronteira ao Norte: face $n$

$$
\begin{aligned}
T_{i, m_{2}-1}^{n+1}= & (1-2 \alpha-3 \lambda) T_{i, m_{2}-1}^{n}+ \\
& \alpha\left(T_{i+1, m_{2}-1}^{n}+T_{i-1, m_{2}-1}^{n}\right)+ \\
& \lambda\left(2 T_{i, m_{2}-\frac{1}{2}}^{n}+T_{i, m_{2}-2}^{n}\right)+ \\
& \frac{\Delta t}{c \rho} f_{i, m_{2}-1}^{n}
\end{aligned}
$$

com $i=2:\left(m_{1}-2\right)$.

- Fronteira ao Sul: face $s$

$$
\begin{aligned}
T_{i, 1}^{n+1}= & (1-2 \alpha-3 \lambda) T_{i, 1}^{n}+ \\
& \alpha\left(T_{i+1,1}^{n}+T_{i-1,1}^{n}\right)+\lambda\left(T_{i, 2}^{n}+T_{i, \frac{1}{2}}^{n}\right)+ \\
& \frac{\Delta t}{c \rho} \tilde{f}_{i, 1}^{n}
\end{aligned}
$$

com $i=2:\left(m_{1}-2\right)$.

No caso do volume de controle ter duas faces na fronteira, por exemplo, como o da Figura 9, as condições de contorno são inseridas pelos termos $\left.\left(k_{x} \frac{\partial T}{\partial x}\right)\right|_{c w} \mathrm{e}$ $\left.\left(k_{x} \frac{\partial T}{\partial x}\right)\right|_{\mathcal{C S}}$, de maneira análoga à dos casos anteriores.

Assim, as formas discretizadas para a equação (9) são as seguintes:

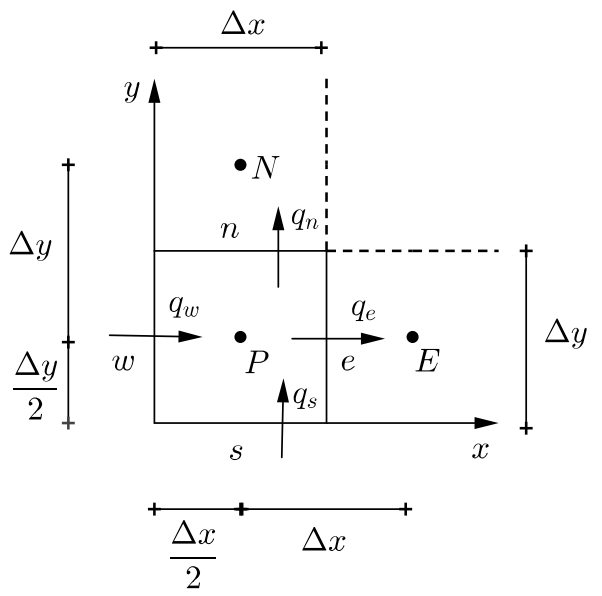

Figura 9: Volume com duas faces na fronteira

- Volume de fronteira nas faces $w$ e $s$

$$
\begin{aligned}
T_{1,1}^{n+1}= & {[1-3(\alpha+\lambda)] T_{1,1}^{n}+\alpha\left(T_{2,1}^{n}+2 T_{\frac{1}{2}, 1}^{n}\right)+} \\
& \lambda\left(T_{1,2}^{n}+2 T_{1, \frac{1}{2}}^{n}\right)+\frac{\Delta t}{c \rho} f_{1,1}^{n} .
\end{aligned}
$$

- Volume de fronteira nas faces $e$ e $s$

$$
\begin{aligned}
T_{m_{1}-1,1}^{n+1}= & {[1-3(\alpha+\lambda)] T_{m_{1}-1,1}^{n}+} \\
& \alpha\left(2 T_{m_{1}-\frac{1}{2}, 1}^{n}+T_{m_{1}-2,1}^{n}\right)+ \\
& \lambda\left(T_{m_{1}-1,2}^{n}+2 T_{m_{1}-1, \frac{1}{2}}^{n}\right)+ \\
& \frac{\Delta t}{c \rho} f_{m_{1}-1,1}^{n} .
\end{aligned}
$$

- Volume de fronteira nas faces $w$ e $n$

$$
\begin{aligned}
T_{1, m_{2}-1}^{n+1}= & {[1-3(\alpha+\lambda)] T_{1, m_{2}-1}^{n}+} \\
& \alpha\left(T_{2, m_{2}-1}^{n}+2 T_{\frac{1}{2}, m_{2}-1}^{n}\right)+ \\
& \lambda\left(2 T_{1, m_{2}-\frac{1}{2}}^{n}+T_{1, m_{2}-2}^{n}\right)+ \\
& \frac{\Delta t}{c \rho} f_{1, m_{2}-1}^{n} .
\end{aligned}
$$

- Volume de fronteira nas faces $e$ e $n$

$$
\begin{aligned}
T_{m_{1}-1, m_{2}-1}^{n+1}= & {[1-3(\alpha+\lambda)] T_{m_{1}-1, m_{2}-1}^{n}+} \\
& \alpha\left(2 T_{m_{1}-\frac{1}{2}, m_{2}-1}^{n}+T_{m_{1}-2, m_{2}-1}^{n}\right)+ \\
& \lambda\left(2 T_{m_{1}-1, m_{2}-\frac{1}{2}}^{n}+T_{m_{1}-1, m_{2}-2}^{n}\right)+ \\
& \frac{\Delta t}{c \rho} f_{m_{1}-1, m_{2}-1}^{n} .
\end{aligned}
$$




\section{Experimento Computacional}

Neste experimento aplicou-se os métodos Explícito, Implícito e de Crank-Nicolson para encontrar aproximações para a equação do calor

$$
\frac{\partial T}{\partial t}-\left(\frac{\partial^{2} T}{\partial x^{2}}+\frac{\partial^{2} T}{\partial y^{2}}\right)=0
$$

com $0 \leq y \leq 1,0 \leq x \leq 1$ e $t \geq 0$, à qual adicionou-se as condições iniciais e de contorno:

$$
\begin{aligned}
T(0, y, t) & =T(1, y, t)=0 \\
T(x, 0, t) & =T(x, 1, t)=0 \\
T(x, y, 0) & =\operatorname{sen}(\pi x) \operatorname{sen}(2 \pi y)
\end{aligned}
$$

Por derivação nas variáveis $t, x$ e $y$ verifica-se diretamente que

$$
T_{e}(x, y, t)=e^{-5 \pi^{2} t} \operatorname{sen}(\pi x) \operatorname{sen}(2 \pi y)
$$

satisfaz a equação diferencial e as condições adicionais do problema. Para encontrar soluções aproximadas com os métodos Explícito, Implícito e de Crank-Nicolson, aplicadas neste caso em particular, utilizou-se o software Matlab.

Na Tabela 1, apresenta-se o erro correspondente ao método explícito no tempo $\mathrm{t}=0.1$ para $N$ pontos da malha nas direções $x$ e $y$. Para obter este resultado usamos $\Delta x=\Delta y=\frac{1}{N}$ e $\Delta t=0,8 \cdot 10^{-4}$ para $N=51$. Esta Tabela também indica os erros correspondentes aos métodos implícitos e de Crank-Nicolson, considerando $\Delta t=0.005$ também para $N=51$ e no tempo $t=0.1$.

Tabela 1: Erros das aproximações

\begin{tabular}{llccc}
\hline & $t$ & $N$ & $\mathrm{~N}^{o}$ de Passos & Erros \\
\hline Explicito & 0.1 & 51 & 1250 & $0,4 \cdot 10^{-4}$ \\
\hline Implícito & 0.1 & 51 & 20 & 0.2004 \\
\hline Crank-Nicolson & 0.1 & 51 & 20 & 0.0461 \\
\hline
\end{tabular}

Adotou-se como medida de erros na Tabelas 1,

$$
\operatorname{Erro}(T)=\left[\sum_{i j}\left(T_{i j}-T_{e}\right)^{2}\right]^{\frac{1}{2}} .
$$

As Figuras 10, 11 e 12 mostram os resultados das aproximações para a equação (35) correspondentes aos métodos Explícitos, Implícitos e de Crank-Nicolson, respectivamente, para $N=51$. Para comparação apresentase também na Figura 13, o gráfico da solução exata.

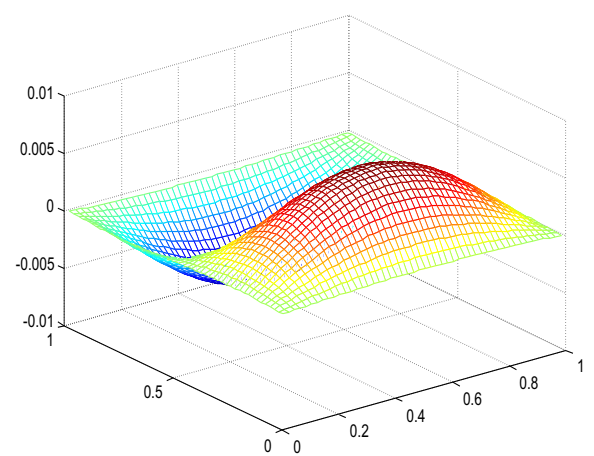

Figura 10: Aproximação do método explícito

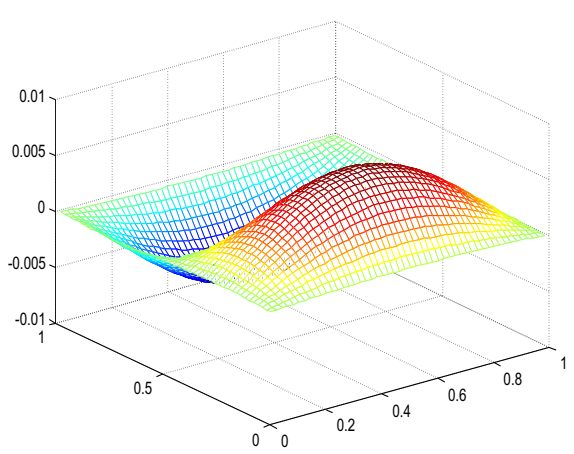

Figura 11: Aproximação do método implícito

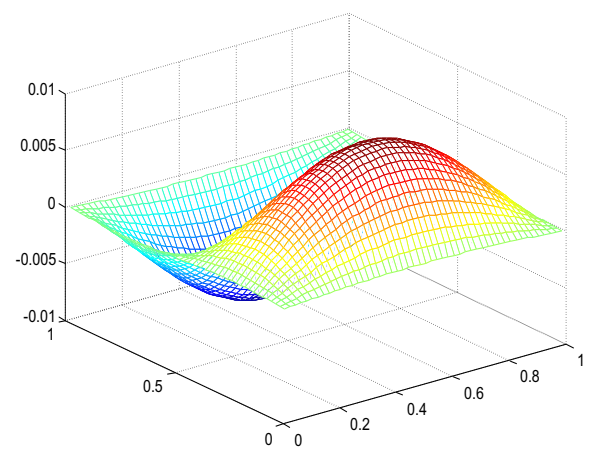

Figura 12: Aproximação do método de CranK- Nicolson

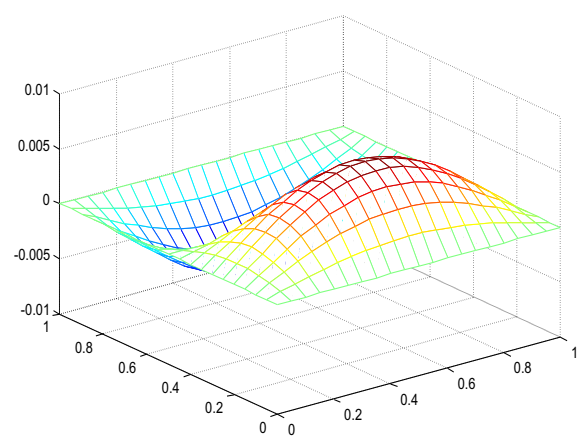

Figura 13: Solução exata 


\section{Conclusões}

No presente estudo, encontra-se resultados significativos para aproximações da solução da equação do calor transiente e bidimensional. Tais resultados foram obtidos utilizando o método de volumes finitos, usando uma malha estruturada; a fronteira do domínio coincide com as linhas coordenadas, facilitando o trabalho de discretização e de aplicação das condições de contorno. Todavia, o método é aplicável em malhas não-estruturadas e em domínios com contornos arbitrários, de acordo com Maliska (2004).

Segundo Ertekin et al. (2001), outros problemas que envolvem equações diferenciais parciais podem ser estudados utilizando esta ferramenta. Em particular, esta técnica pode ser usada para encontrar a aproximação da solução para problemas complexos em várias áreas da ciência e da engenharia. Como por exemplo, pode-se avaliar a eficiência da recuperação do petróleo disponível num reservatório.

\section{Referências}

Cunha, M. C. C. (2003). Métodos Numéricos, $2^{\circ}$ edn. Editora da Unicamp.

Ertekin, T., Abou-Kassem, J. H., King, G. R. (2001). Basic Applied Reservoir Simulation. Richardson, TX: Society of Petroleum Engineers.

Kreider, D. L. (1972). Introdução à análise linear. Ao Livro Tecnico.

Lin, C., Segel, L. A. (1974). Mathematics Applied to Deterministic Problems in the Natural Sciences. SIAM.

Maliska, C. R. (2004). Transferência de calor e mecânica dos fluidos computacionais, $2^{\circ}$ edn. Livros Técnicos e Científicos.

de Oliveira Fortuna, A. (2000). Técnicas computacionais para dinâmica dos fluidos: Conceitos Básicos e Aplicações. Edusp.

Sperandio, D., Mendes, J. T., e Silva, L. H. M. (2003). Cálculo numérico: características matemáticas e computacionais dos métodos numéricos. Prentice Hall.

Thomas, J. W. (1995). Numerical partial differential equations: finite difference methods. Springer. 